\title{
Quality of Life in Patients with Papillary Thyroid Microcarcinoma According to Treatment: Total Thyroidectomy with or without Radioactive Iodine Ablation
}

\author{
Jonghwa Ahn, Min Ji Jeon, Eyun Song, Tae Yong Kim, Won Bae Kim, Young Kee Shong, Won Gu Kim \\ Department of Internal Medicine, Asan Medical Center, University of Ulsan College of Medicine, Seoul, Korea
}

Background: Recently, there has been some controversy regarding the role of radioactive iodine (RAI) ablation in the treatment of low-risk differentiated thyroid carcinoma (DTC), especially papillary thyroid microcarcinoma (PTMC). This study aimed to compare quality of life (QoL) parameters between patients with PTMC who underwent total thyroidectomy (TT) alone and those who underwent TT with RAI ablation.

Methods: In this cross-sectional study, patients with PTMC who underwent TT with/without RAI remnant ablation were prospectively enrolled between June 2016 and October 2017. All patients completed three questionnaires: the 12-item short-form health survey (SF-12), thyroid cancer-specific quality of life (THYCA-QoL) questionnaire, and fear of progression (FoP) questionnaire.

Results: The TT and TT with RAI groups comprised 107 and 182 patients, respectively. The TT with RAI group had significantly lower serum thyrotropin (TSH) levels than the TT group. However, after matching for TSH levels between the groups $(n=100$ in both groups), there were no significant differences in baseline characteristics. According to the SF-12, the score for general health was significantly lower in the TT with RAI group than in the TT group $(P=0.047)$. The THYCA-QoL also showed a significant difference in the "felt chilly" score between groups $(P=0.023)$. No significant differences in FoP scores were observed between the groups.

Conclusion: Patients with PTMC who underwent TT with RAI ablation experienced more health-related problems than those managed with TT alone. These findings support the idea that RAI ablation should be carefully considered in patients with low-risk DTCs.

Keywords: Quality of life; Radioactive iodine; Thyroidectomy; Papillary thyroid microcarcinoma

\section{INTRODUCTION}

As imaging technology has advanced, the incidence of differentiated thyroid carcinoma (DTC) diagnosis has increased. Most DTCs are microcarcinomas, defined as cancers $1 \mathrm{~cm}$ or less in size $[1,2]$. The traditional treatment for DTC is total thyroidec-

Received: 13 November 2019, Revised: 26 December 2019,

Accepted: 31 December 2019

Corresponding author: Won Gu Kim

Division of Endocrinology and Metabolism, Department of Internal Medicine,

Asan Medical Center, University of Ulsan College of Medicine, 88 Olympic-ro

43-gil, Songpa-gu, Seoul 05505, Korea

Tel: +82-2-3010-5883, Fax: +82-2-3010-6962

E-mail: wongukim@amc.seoul.kr tomy (TT) followed by radioactive iodine (RAI) ablation and thyrotropin (TSH) suppression [3]. The goals of RAI ablation after TT are to remove remnant thyroid tissues, including microscopic thyroid cancer; to allow for the evaluation of the evidence for metastatic disease using whole-body scanning; to enable risk stratification and TSH suppression therapy; and to al-

\section{Copyright $\odot 2020$ Korean Endocrine Society}

This is an Open Access article distributed under the terms of the Creative Commons Attribution Non-Commercial License (https://creativecommons.org/ licenses/by-nc/4.0/) which permits unrestricted non-commercial use, distribution, and reproduction in any medium, provided the original work is properly cited. 
low for the thyroglobulin level to be used as a predictor of recurrence $[1,4]$. However, RAI ablation may cause fatigue, edema, sialadenitis, xerostomia, second primary malignancies, and other side effects [4]. According to the American Thyroid Association risk stratification guidelines, low-risk DTCs and papillary thyroid microcarcinomas (PTMCs) generally have excellent prognoses $[5,6]$, and the role of RAI ablation in the treatment of PTMCs and low-risk DTCs is controversial $[1,7,8]$.

Assessing the quality of life (QoL) in patients with thyroid cancer might be helpful in determining appropriate treatment strategies, especially RAI therapy [9-11]. Several studies have evaluated QoL in patients with DTC $[9,10,12,13]$. The prognoses of patients with DTC were excellent, but their QoL was poor compared with that of the general population [10,12,14]. RAI therapy may be the reason for this poor QoL in DTC survivors. To the best of our knowledge, no studies have compared QoL between patients who underwent TT alone and those who underwent TT with RAI. Therefore, this study aimed to compare QoL parameters between patients with PTMC who underwent TT alone and those who underwent TT with RAI ablation.

\section{METHODS}

\section{Patients}

Patients with PTMC who underwent TT with/without RAI remnant ablation between June 2016 and October 2017 were prospectively enrolled in this cross-sectional study. During their regular visits, study was fully explained and written consent was obtained. Patients then completed questionnaires, described below. Patients who were previously diagnosed with psychiatric disorders, rheumatologic disorders, and other cancers were excluded. Furthermore, patients with uncontrolled chronic, those requiring three or more medications for hypertension control, and those requiring insulin injection for diabetes control were excluded. We collected demographic characteristics such as age, sex, marital status, education level, employment status, and socioeconomic status that can affect QoL. Medical records and clinical laboratory data such as serum TSH level at the time of questionnaire completion were reviewed and collected. The study protocol was approved by the Institutional Review Board of Asan Medical Center (study number: 2016-0606) and was registered with the Clinical Research Information Service at the Korea Centers for Disease Control and Prevention (KCT0001986) [9].

\section{The RAI dose}

RAI remnant ablation was conducted according to Asan Medi- cal Center protocols. PTMC without extrathyroidal extension (ETE) and cervical lymph node (LN) metastasis (pathologically N0) could be observed without RAI or treated with $30 \mathrm{mCi}$ of RAI. PTMC with microscopic ETE and N0 was treated with 30 $\mathrm{mCi}$ of RAI. PTMC with central cervical LN metastasis (pathologically N1a) was treated with $80 \mathrm{mCi}$ of RAI.

\section{Questionnaires}

To assess QoL, all patients completed three questionnaires: the 12-item short-form health survey (SF-12), thyroid cancer-specific quality of life (THYCA-QoL) questionnaire, and fear of progression (FoP) questionnaire. The SF-12 (v2.0) is a well-validated short-form of SF-36 that consists of 12 questions that provide an eight-dimensional profile of health status, including physical functioning, role limitations due to physical health, role limitations due to emotional problems, general health, vitality, bodily pain, social functioning, and mental health. Answers were combined and weighted to produce Mental and Physical Component Summary scores using the Quality Metric Health Outcomes Scoring Software version 5.0 (QualityMetric, Lincoln, RI, USA) (range, 0 to 100). Lower scores indicated a worse health status [15]. The THYCA-QoL is the first THYCAQoL questionnaire and contains 24 items that assess symptoms from thyroid cancer or its treatment. Each item was scored on a four-point response scale ranging from 1, "not at all" to 4, "very much." Each score was converted into scores on seven multisymptom scales and six single-symptom scales. A lower score indicated lesser complaints caused by that symptom $[16,17]$. FoP evaluates anxiety and fear of disease progression using a 43-item questionnaire. Each item belongs to five subcategories: affective reactions (13 items), partnership/family (seven items), work (seven items), loss of autonomy (seven items), and coping with anxiety (nine items). In all subcategories except coping with anxiety, a higher score indicated more anxiety about disease progression. Conversely, in the coping with anxiety subcategory, a higher score indicated better coping [18].

\section{Statistics}

R studio version 3.1.3 (R Foundation for Statistical Computing, Vienna, Austria) was used for data analysis. Continuous variables are presented as means and standard deviations or as medians and interquartile ranges (IQR). Categorical variables are presented as numbers with percentages. We used the $t$ test and Wilcoxon's test to compare continuous variables and the chi-square test to compare categorical variables. Because serum TSH values only show normal distribution after log transformation, serum 
TSH values were log-transformed, and the geometric mean values were analyzed. All $P$ values were two-sided, and a $P$ value less than 0.05 was considered statistically significant [9].

\section{RESULTS}

\section{Baseline characteristics}

Baseline characteristics of 289 PTMC patients who underwent TT with/without RAI are shown in Table 1. Most patients (99\%, $n=287$ ) underwent TT with central node dissection. The TT alone and TT with RAI groups included 107 and 182 patients, respectively. The median age of the patients was mid-50s, and most patients were female. No patients had structural disease at the time of questionnaire completion. The median time intervals between TT and questionnaire completion in the TT alone and TT with RAI groups were 3.5 years (IQR, 2.4 to 4.8 ) and 3.8 years (IQR, 2.9 to 4.5$)$, respectively $(P=0.66)$.

Table 2 shows the pathologic findings and the RAI dose that patients received. None of the patients in the TT alone group had cervical LN metastasis. In contrast, 106 patients (58\%) in the TT and RAI group had cervical LN metastasis (pathologically N1a). In the TT and RAI group, 72 patients (40\%) received $30 \mathrm{mCi}$ of RAI and $110(60 \%)$ received $80 \mathrm{mCi}$ of RAI owing to the presence of microscopic ETE or cervical LN metastasis. The median dose of RAI was $80 \mathrm{mCi}$. In terms of preparation for RAI, 40 patients (22\%) received recombinant human thyrotropin (rhTSH) rather than undergoing thyroid hormone withdrawal. The median interval between ablation and questionnaire completion was 3.7 years (IQR, 2.8 to 4.4 ). No patients underwent repeated RAI treatment.

Compared with patients in the TT alone group, those in the TT with RAI group were more frequently married $(P=0.031)$ and exhibited lower serum TSH levels $(P<0.001)$. After match- ing for TSH levels, the TT alone and TT with RAI groups each comprised 100 patients (Fig. 1). There were no significant differences in baseline characteristics between the two post-matching groups (Table 1).

No patients have subsequently presented with disease recurrence, and no deaths were reported as of the last follow-up date (November 31, 2019).

\section{QoL analysis}

Scores for QoL parameters from the three questionnaires are shown in Table 3. Among the SF-12 parameters, the scores for general health in the TT alone and TT with RAI groups were significantly different between the TSH-matched cohorts (49.96 vs. 48.05, $P=0.047$ ), which suggested poorer health status in the TT with RAI group. The "felt chilly" parameter in the THYCA-QoL also showed a significant difference between the matched groups $(P=0.023)$, which suggested more chilly feelings in patients in the TT with RAI group. There were no significant differences in FoP scores between the groups.

In the TT with RAI group, no significant differences in QoL scores were observed between patients prepared for treatment with thyroid hormone withdrawal versus those treated with rhTSH. There was also no significant difference in the QoL parameters according to the RAI dose (data not shown).

\section{DISCUSSION}

We evaluated QoL in PTMC patients who underwent TT with/ without RAI using three well-validated questionnaires. After matching for TSH levels, patients who underwent TT with RAI presented lower scores for QoL parameters such as "general health" in the SF-12 and "felt chilly" in the THYCA-QoL, although their TSH level was within the reference range. These

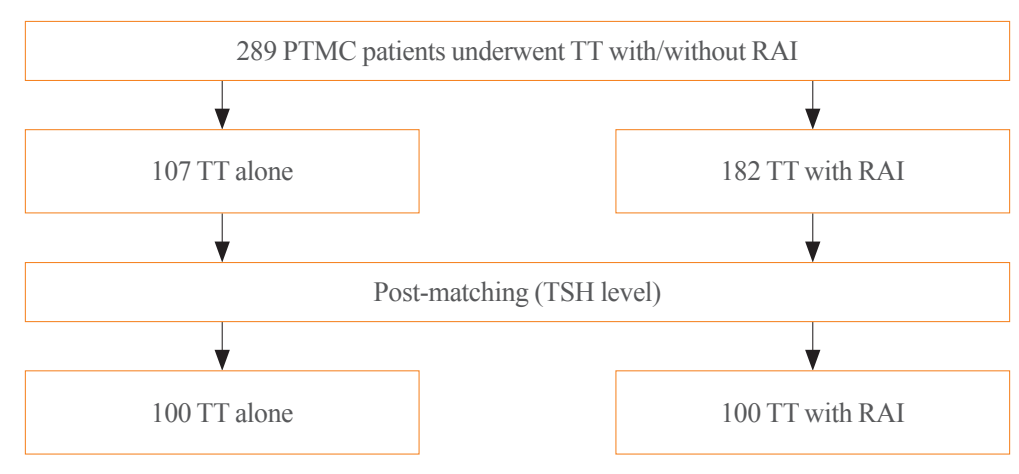

Fig. 1. Papillary thyroid microcarcinoma (PTMC) patients were grouped by treatment type and matched by thyrotropin (TSH) levels for comparisons between groups. TT, total thyroidectomy; RAI, radioactive iodine remnant ablation. 
Table 1. Baseline Characteristics of Patients with Papillary Thyroid Microcarcinoma

\begin{tabular}{|c|c|c|c|c|c|c|}
\hline \multirow{2}{*}{ Characteristic } & \multicolumn{3}{|c|}{ Total population } & \multicolumn{3}{|c|}{ Post-matching $^{\mathrm{a}}$} \\
\hline & TT only $(n=107)$ & TT-RAI $(n=182)$ & $P$ value & TT only $(n=100)$ & TT-RAI $(n=100)$ & $P$ value \\
\hline Age, yr & $54.24 \pm 8.99$ & $52.91 \pm 9.47$ & 0.24 & $54.13 \pm 8.71$ & $52.87 \pm 9.68$ & 0.33 \\
\hline Female sex & $96(90)$ & $150(82)$ & 0.13 & $93(93)$ & $83(83)$ & 0.14 \\
\hline \multicolumn{7}{|l|}{ Marital status } \\
\hline Married & $86(80)$ & $164(90)$ & 0.031 & $83(83)$ & $90(90)$ & 0.21 \\
\hline \multicolumn{7}{|l|}{ Education level } \\
\hline College degree or higher & $40(37)$ & $86(47)$ & 0.13 & $39(39)$ & $43(43)$ & 0.67 \\
\hline \multicolumn{7}{|l|}{ Current employment status } \\
\hline Employed & $46(43)$ & $90(49)$ & 0.34 & $42(42)$ & $51(51)$ & 0.26 \\
\hline Socioeconomic status & & & 0.11 & & & 0.07 \\
\hline Upper & $49(46)$ & $64(35)$ & & $45(45)$ & $31(31)$ & \\
\hline Middle & $40(37)$ & $92(50)$ & & $39(39)$ & $55(55)$ & \\
\hline Lower & $18(17)$ & $27(15)$ & & $16(16)$ & $14(14)$ & \\
\hline Serum TSH, mIU/L $\mathrm{L}^{\mathrm{b}}$ & $0.35 \pm 4.85$ & $0.16 \pm 4.39$ & $<0.001$ & $0.30 \pm 4.34$ & $0.25 \pm 4.42$ & 0.38 \\
\hline
\end{tabular}

Values are expressed as mean \pm standard deviation or number (\%).

TT, total thyroidectomy; RAI, radioactive iodine; TSH, thyrotropin.

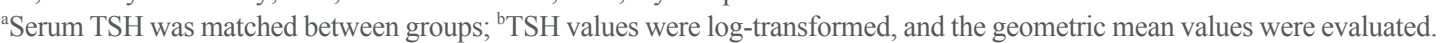

Table 2. Pathologic Findings and Radioactive Iodine Dose of the Patients

\begin{tabular}{lcc}
\hline Variable & TT only $(n=107)$ & TT-RAI $(n=182)$ \\
\hline TNM stage & $107(100)$ & $182(100)$ \\
T1 & $2(1.9)$ & $116(63.4)$ \\
Microscopic ETE & $107(100)$ & $76(41.8)$ \\
N0 & - & $106(58.2)$ \\
N1a & $107(100)$ & $182(100)$ \\
M0 & & $142(78.0)$ \\
RAI preparation & - & $40(22.0)$ \\
T4 withdrawal & - & $72(39.6)$ \\
rhTSH & - & $110(60.4)$ \\
RAI dose & - &
\end{tabular}

Values are expressed as number (\%).

TT, total thyroidectomy; RAI, radioactive iodine; TNM, tumor node metastasis according to the eighth edition of the American Joint Committee on Cancer/Union for International Cancer Control; ETE, extrathyroidal extension; T4, thyroxine; rhTSH, recombinant human thyrotropin.

results suggested that RAI therapy could negatively affect QoL in patients with thyroid cancer. In low-risk DTC or PTMC, RAI remnant ablation has not been shown to benefit clinical out- comes $[7,8,19,20]$. Therefore, weighing the risks and benefits of RAI is important. Because DTC has an excellent prognosis and DTC patients live for a long time, efforts to preserve QoL after treatment are needed. Our study suggests that RAI remnant ablation might cause harm in terms of QoL in patients with PTMC.

Previously, little was known about QoL in patients with DTC, especially with respect to RAI. QoL in patients with thyroid cancer is shown to be poorer than that in the general population $[21,22]$. In a previous study that evaluated QoL using the SF-36 and other questionnaires, patients who underwent TT with RAI had lower social functioning and general health perception than healthy controls [23]. For RAI, patients need to stop hormone replacement for several weeks if they are not taking recombinant TSH. This can lead to physical and emotional instability in patients, and there is evidence of reduced glomerular filtration rate and liver enzyme elevation. With the use of recombinant TSH instead of hormone withdrawal, the discomfort and complications of hypothyroidism during the pre-ablation period are reduced [21]. However, RAI can cause multiorgan complications that can occur at various times [4]. Side effects related to the salivary glands, such as sialadenitis and xerostomia, were the most frequent complications of RAI. RAI also affected the eye and gastrointestinal systems in the early-to-intermediate phase of treatment. Although occurrences were rare, hematologic complications such as aplastic anemia and leukemia were 
Table 3. Quality of Life Parameters According to Treatment

\begin{tabular}{|c|c|c|c|c|c|c|}
\hline \multirow{2}{*}{ Variable } & \multicolumn{3}{|c|}{ Total population } & \multicolumn{3}{|c|}{ Post-matching $^{a}$} \\
\hline & TT only $(n=107)$ & TT-RAI $(n=182)$ & $P$ value & TT only $(n=100)$ & TT-RAI $(n=100)$ & $P$ value \\
\hline \multicolumn{7}{|l|}{ SF-12 v2 } \\
\hline PCS & $50.44 \pm 6.50$ & $50.16 \pm 6.81$ & 0.72 & $50.83 \pm 6.45$ & $49.40 \pm 6.97$ & 0.13 \\
\hline Physical functioning & $49.19 \pm 8.72$ & $49.50 \pm 8.15$ & 0.76 & $49.74 \pm 8.16$ & $48.11 \pm 8.21$ & 0.16 \\
\hline Role-physical & $46.94 \pm 7.54$ & $47.12 \pm 8.06$ & 0.86 & $47.05 \pm 7.55$ & $46.46 \pm 7.86$ & 0.59 \\
\hline Bodily pain & $52.25 \pm 6.98$ & $51.78 \pm 8.23$ & 0.62 & $52.32 \pm 6.66$ & $51.33 \pm 8.04$ & 0.34 \\
\hline General health & $49.96 \pm 6.33$ & $48.82 \pm 7.30$ & 0.18 & $49.96 \pm 6.19$ & $48.05 \pm 7.29$ & $<0.05$ \\
\hline MCS & $48.14 \pm 9.24$ & $48.13 \pm 8.96$ & 0.99 & $47.83 \pm 9.24$ & $47.50 \pm 8.34$ & 0.78 \\
\hline Vitality & $49.71 \pm 10.92$ & $49.55 \pm 10.20$ & 0.90 & $49.56 \pm 11.04$ & $48.97 \pm 9.93$ & 0.69 \\
\hline Social functioning & $51.91 \pm 8.57$ & $51.77 \pm 7.94$ & 0.86 & $52.28 \pm 7.94$ & $51.74 \pm 7.81$ & 0.63 \\
\hline Role-emotional & $45.16 \pm 9.93$ & $44.80 \pm 10.73$ & 0.78 & $45.05 \pm 9.82$ & $43.44 \pm 10.22$ & 0.26 \\
\hline Mental health & $48.88 \pm 9.08$ & $49.40 \pm 9.01$ & 0.64 & $48.49 \pm 9.25$ & $48.66 \pm 8.94$ & 0.89 \\
\hline \multicolumn{7}{|l|}{ THYCA-QoL } \\
\hline Neuromuscular & $48.21 \pm 15.40$ & $49.73 \pm 16.08$ & 0.43 & $48.08 \pm 14.60$ & $50.83 \pm 15.51$ & 0.20 \\
\hline Voice & $37.73 \pm 14.36$ & $37.84 \pm 16.69$ & 0.95 & $37.75 \pm 14.10$ & $38.25 \pm 15.67$ & 0.81 \\
\hline Concentration & $41.59 \pm 16.45$ & $38.94 \pm 16.00$ & 0.18 & $40.50 \pm 15.30$ & $39.88 \pm 16.35$ & 0.78 \\
\hline Sympathetic & $44.16 \pm 17.62$ & $45.28 \pm 19.13$ & 0.62 & $43.75 \pm 17.18$ & $45.91 \pm 19.19$ & 0.40 \\
\hline Throat/mouth & $38.40 \pm 10.60$ & $39.84 \pm 13.84$ & 0.32 & $38.42 \pm 10.65$ & $40.25 \pm 12.37$ & 0.26 \\
\hline Psychological & $44.04 \pm 13.61$ & $44.54 \pm 14.47$ & 0.77 & $43.94 \pm 12.79$ & $45.69 \pm 13.75$ & 0.35 \\
\hline Sensory & $48.13 \pm 19.10$ & $49.18 \pm 18.33$ & 0.65 & $47.88 \pm 18.97$ & $50.00 \pm 18.63$ & 0.43 \\
\hline Problems with scar & $38.55 \pm 21.52$ & $35.85 \pm 19.20$ & 0.27 & $38.25 \pm 20.86$ & $36.25 \pm 19.26$ & 0.48 \\
\hline Felt chilly & $34.58 \pm 15.22$ & $38.60 \pm 19.05$ & 0.05 & $33.50 \pm 13.40$ & $38.75 \pm 19.59$ & $<0.05$ \\
\hline Tingling hands/feet & $48.83 \pm 17.97$ & $50.41 \pm 20.60$ & 0.51 & $48.25 \pm 17.86$ & $51.00 \pm 20.08$ & 0.31 \\
\hline Gained weight & $44.86 \pm 24.46$ & $43.96 \pm 22.40$ & 0.75 & $45.00 \pm 24.36$ & $47.75 \pm 23.33$ & 0.42 \\
\hline Headache & $40.65 \pm 17.69$ & $42.58 \pm 19.49$ & 0.40 & $40.50 \pm 17.69$ & $42.50 \pm 18.97$ & 0.44 \\
\hline Less interest in sex & $42.06 \pm 18.04$ & $42.86 \pm 18.50$ & 0.72 & $42.25 \pm 17.30$ & $40.75 \pm 16.54$ & 0.53 \\
\hline \multicolumn{7}{|l|}{ Fear of progression } \\
\hline Affective reactions & $1.93 \pm 0.71$ & $1.95 \pm 0.83$ & 0.86 & $1.94 \pm 0.70$ & $1.96 \pm 0.81$ & 0.80 \\
\hline Partnership/family & $1.83 \pm 0.68$ & $1.93 \pm 0.92$ & 0.30 & $1.84 \pm 0.69$ & $1.90 \pm 0.73$ & 0.53 \\
\hline Work & $1.53 \pm 0.72$ & $1.51 \pm 0.75$ & 0.87 & $1.51 \pm 0.73$ & $1.44 \pm 0.65$ & 0.48 \\
\hline Loss of autonomy & $1.58 \pm 0.62$ & $1.61 \pm 0.71$ & 0.76 & $1.59 \pm 0.63$ & $1.61 \pm 0.67$ & 0.84 \\
\hline Coping with anxiety & $2.89 \pm 0.83$ & $3.06 \pm 0.94$ & 0.13 & $2.89 \pm 0.81$ & $3.08 \pm 0.97$ & 0.13 \\
\hline
\end{tabular}

Values are expressed as mean \pm standard deviation.

TT, total thyroidectomy; RAI, radioactive iodine; SF-12, 12-item short-form health survey; PCS, physical component summary score; MCS, mental component summary score; THYCA-QoL, thyroid cancer-specific quality of life.

${ }^{\text {a}}$ Serum thyrotropin was matched between groups.

severe. Reduced fertility was also one of the side effects [4]. These complications can occur at any time after RAI and can influence QoL. The reason the "general health" score was lower in the TT with RAI group than in the TT alone group was likely owing to various side effects of RAI. However, because TSH levels in the TT with RAI group were within the reference range, the exact reason for or pathogenesis of the high "felt chilly" score in the TT with RAI group remains unclear. Further research is needed to clarify this mechanism. In terms of the dose, RAI above $5.55 \mathrm{GBq}(150 \mathrm{mCi})$ has been associated with worse QoL [11]. In the present study, the median dose of RAI was $2.96 \mathrm{GBq}(80 \mathrm{mCi})$, but our results showed that even this 
lower dose of RAI could affect QoL. Therefore, the use of RAI in patients with low-risk DTC or PTMC should be carefully considered and should be decided according to individual risk stratification.

QoL impairment due to treatment may improve with time. One study showed that QoL impairment observed during the first year improved over time after treatment [24]. Another study showed that the QoL scores improved according to the duration of cure [23]. In the present study, we showed reduced QoL parameters in the TT with RAI group compared with those in the TT alone group, even after a median period of 3.7 years following initial treatment. This suggests that the effects of RAI remain for a long time. However, further larger prospective multicenter studies need to confirm these results.

There were some limitations in this study. First, this was a cross-sectional study, in which the questionnaires were conducted at one timepoint without follow-up. Therefore, it is hard to evaluate changes in QoL after treatment, and the results could be influenced by confounding factors. However, to minimize this, we attempted to match TSH levels in both groups, and there were no significant differences in baseline characteristics that could affect QoL. Second, the number of patients was small, which could affect the statistical power. Despite these limitations, this is the first study to demonstrate the effect of RAI on QoL by comparing the TT alone and TT with RAI groups.

In conclusion, patients with PTMC who underwent TT with RAI ablation experienced more health-related problems than those managed by TT alone. This effect was present even after a median period of 4 years since initial therapy and the maintenance of euthyroid status. These findings support the idea that RAI ablation should be carefully considered in patients with low-risk DTCs because it could negatively affect their QoL.

\section{CONFLICTS OF INTEREST}

No potential conflict of interest relevant to this article was reported.

\section{ACKNOWLEDGMENTS}

This study was supported by the Korean Endocrine Society with the Korean Endocrine Society Research Award 2018.

\section{AUTHOR CONTRIBUTIONS}

Conception or design: M.J.J., T.Y.K., W.B.K., Y.K.S., W.G.K.
Acquisition, analysis, or interpretation of data: J.A., E.S. Drafting the work or revising: J.A., M.J.J., W.G.K. Final approval of the manuscript: J.A., M.J.J., T.Y.K., W.B.K., Y.K.S., W.G.K.

\section{ORCID}

Jonghwa Ahn https://orcid.org/0000-0002-7592-3585

Won Gu Kim https://orcid.org/0000-0002-8404-7759

\section{REFERENCES}

1. Haugen BR. 2015 American Thyroid Association management guidelines for adult patients with thyroid nodules and differentiated thyroid cancer: what is new and what has changed? Cancer 2017;123:372-81.

2. Ahn HS, Kim HJ, Welch HG. Korea's thyroid-cancer "epidemic": screening and overdiagnosis. N Engl J Med 2014; 371:1765-7.

3. American Thyroid Association (ATA) Guidelines Taskforce on Thyroid Nodules and Differentiated Thyroid Cancer, Cooper DS, Doherty GM, Haugen BR, Kloos RT, Lee SL, et al. Revised American Thyroid Association management guidelines for patients with thyroid nodules and differentiated thyroid cancer. Thyroid 2009;19:1167-214.

4. Van Nostrand D. The benefits and risks of I-131 therapy in patients with well-differentiated thyroid cancer. Thyroid 2009;19:1381-91.

5. Jeon MJ, Kim WG, Choi YM, Kwon H, Lee YM, Sung TY, et al. Features predictive of distant metastasis in papillary thyroid microcarcinomas. Thyroid 2016;26:161-8.

6. Jeon MJ, Kim WG, Kwon H, Kim M, Park S, Oh HS, et al. Clinical outcomes after delayed thyroid surgery in patients with papillary thyroid microcarcinoma. Eur J Endocrinol 2017;177:25-31.

7. Schvartz C, Bonnetain F, Dabakuyo S, Gauthier M, Cueff A, Fieffe $\mathrm{S}$, et al. Impact on overall survival of radioactive iodine in low-risk differentiated thyroid cancer patients. J Clin Endocrinol Metab 2012;97:1526-35.

8. Sacks W, Fung CH, Chang JT, Waxman A, Braunstein GD. The effectiveness of radioactive iodine for treatment of lowrisk thyroid cancer: a systematic analysis of the peer-reviewed literature from 1966 to April 2008. Thyroid 2010;20: 1235-45.

9. Jeon MJ, Lee YM, Sung TY, Han M, Shin YW, Kim WG, et al. Quality of life in patients with papillary thyroid microcarcinoma managed by active surveillance or lobectomy: a 
cross-sectional study. Thyroid 2019;29:956-62.

10. Husson O, Haak HR, Buffart LM, Nieuwlaat WA, Oranje WA, Mols F, et al. Health-related quality of life and disease specific symptoms in long-term thyroid cancer survivors: a study from the population-based PROFILES registry. Acta Oncol 2013;52:249-58.

11. Almeida JP, Vartanian JG, Kowalski LP. Clinical predictors of quality of life in patients with initial differentiated thyroid cancers. Arch Otolaryngol Head Neck Surg 2009;135:3426.

12. Goldfarb M, Casillas J. Thyroid cancer-specific quality of life and health-related quality of life in young adult thyroid cancer survivors. Thyroid 2016;26:923-32.

13. Sung TY, Shin YW, Nam KH, Chang HS, Rhee Y, Park CS, et al. Psychological impact of thyroid surgery on patients with well-differentiated papillary thyroid cancer. Qual Life Res 2011;20:1411-7.

14. Husson O, Haak HR, Oranje WA, Mols F, Reemst PH, van de Poll-Franse LV. Health-related quality of life among thyroid cancer survivors: a systematic review. Clin Endocrinol (Oxf) 2011;75:544-54.

15. Ware J Jr, Kosinski M, Keller SD. A 12-item short-form health survey: construction of scales and preliminary tests of reliability and validity. Med Care 1996;34:220-33.

16. Jeong Y, Choi J, Ahn AL, Oh EJ, Oh HK, Cho DY, et al. Validation of the Korean version of the thyroid cancer-specific quality of life questionnaire. Ann Surg Treat Res 2015; 89:287-94.

17. Husson O, Haak HR, Mols F, Nieuwenhuijzen GA, Nieuwlaat WA, Reemst PH, et al. Development of a disease-specific health-related quality of life questionnaire (THYCA-QoL) for thyroid cancer survivors. Acta Oncol 2013;52:447-54.

18. Herschbach P, Berg P, Dankert A, Duran G, Engst-Hastreiter $\mathrm{U}$, Waadt $\mathrm{S}$, et al. Fear of progression in chronic diseases: psychometric properties of the Fear of Progression Questionnaire. J Psychosom Res 2005;58:505-11.

19. Brown RL, de Souza JA, Cohen EE. Thyroid cancer: burden of illness and management of disease. J Cancer 2011;2:1939.

20. Kwon H, Jeon MJ, Kim WG, Park S, Kim M, Kim TY, et al. Lack of efficacy of radioiodine remnant ablation for papillary thyroid microcarcinoma: verification using inverse probability of treatment weighting. Ann Surg Oncol 2017; 24:2596-602.

21. Taieb D, Sebag F, Cherenko M, Baumstarck-Barrau K, Fortanier C, Farman-Ara B, et al. Quality of life changes and clinical outcomes in thyroid cancer patients undergoing radioiodine remnant ablation (RRA) with recombinant human TSH (rhTSH): a randomized controlled study. Clin Endocrinol (Oxf) 2009;71:115-23.

22. Husson O, Nieuwlaat WA, Oranje WA, Haak HR, van de Poll-Franse LV, Mols F. Fatigue among short- and long-term thyroid cancer survivors: results from the population-based PROFILES registry. Thyroid 2013;23:1247-55.

23. Hoftijzer HC, Heemstra KA, Corssmit EP, van der Klaauw AA, Romijn JA, Smit JW. Quality of life in cured patients with differentiated thyroid carcinoma. J Clin Endocrinol Metab 2008;93:200-3.

24. Crevenna R, Zettinig G, Keilani M, Posch M, Schmidinger M, Pirich C, et al. Quality of life in patients with non-metastatic differentiated thyroid cancer under thyroxine supplementation therapy. Support Care Cancer 2003;11:597-603. 